\title{
Diagnostic utility of kidney biopsy in patients with sarcoidosis and acute kidney injury
}

This article was published in the following Dove Press journal: International Journal of Nephrology and Renovascular Disease 23 September 2011

Number of times this article has been viewed

\author{
Ravish Shah' \\ Ganesh Shidham' \\ Anil Agarwal' \\ Alia Albawardi ${ }^{2}$ \\ Tibor Nadasdy ${ }^{2}$ \\ 'Division of Nephrology, ${ }^{2}$ Division \\ of Renal Pathology, The Ohio State \\ University, Columbus, Ohio, USA
}

Correspondence: Ravish Shah The Ohio State University, Division of Nephrology, 395 W I2th Avenue, Ground Floor, Columbus, $\mathrm{OH} 43210$, USA

$\mathrm{Tel}+\mathrm{I} 6 \mid 42934055$

Fax +I 6142933073

Email ravish.shah@osumc.edu
Background: Sarcoidosis is an idiopathic multisystem disease characterized by noncaseating granulomatous inflammation. Renal biopsy is often performed to evaluate the patient with sarcoidosis and acute kidney injury (AKI). Diagnosis rests on the demonstration of noncaseating granulomas and exclusion of other causes of granulomatous inflammation. This paper reports a patient with pulmonary sarcoidosis and AKI whose renal function improved after prednisone therapy despite the absence of kidney biopsy findings characteristic of sarcoidosis.

Case report: A 63-year-old Caucasian male with history of hypertension was treated for pulmonary sarcoidosis with a 6-month course of prednisone. His creatinine was $1.6 \mathrm{mg} / \mathrm{dL}$ during the course. Two months after finishing treatment, he presented with creatinine of $4 \mathrm{mg} / \mathrm{dL}$. A kidney biopsy was performed, which showed nonspecific changes without evidence of granuloma or active interstitial inflammation. He was empirically started on prednisone for presumed renal sarcoidosis, even with a nondiagnostic kidney biopsy finding. Within a month of treatment, his serum creatinine improved to $2 \mathrm{mg} / \mathrm{dL}$, though not to baseline. He continues to be stable on low-dose prednisone. With this case as a background, we aimed to determine the incidence of inconclusive kidney biopsies in patients with sarcoidosis presenting with AKI and to identify the various histological findings seen in this group of patients.

Methods: In this retrospective study, all patients who had native renal biopsies read at The Ohio State University over the period of 6 years were identified. Those patients with a diagnosis of sarcoidosis, presenting with AKI, were included for further review.

Results: Out of 21 kidney biopsies done in patients with sarcoidosis over a period of 6 years, only four (19\%) showed granulomatous interstitial nephritis (GIN). An equal number of patients (4 [19\%]) had presence of membranous nephropathy. Nephrocalcinosis was seen in three patients (14\%). Almost half of the biopsies had findings suggestive of diabetic nephropathy or other nonspecific changes not characteristic of renal sarcoidosis (48\%).

Conclusion: Renal sarcoidosis can be focal in nature and characteristic lesions can be missed in a small-needle core biopsy. Inconclusive renal biopsies with only nonspecific findings are frequent in patients with sarcoidosis and AKI. The presence of GIN on renal biopsy, although classic, is uncommon. Renal sarcoidosis remains a presumptive clinical diagnosis and empiric treatment with steroids may be initiated in cases with a strong clinical suspicion even in the absence of characteristic renal biopsy findings.

Keywords: sarcoidosis, renal sarcoidosis, kidney biopsy, granulomatous interstitial nephritis, acute kidney injury

\section{Background}

Sarcoidosis is an idiopathic multisystem disease characterized by noncaseating granulomatous inflammation. ${ }^{1,2}$ Diagnosis of sarcoidosis rests on the demonstration of 
noncaseating granulomas in a biopsy specimen and clinical exclusion of other causes of granulomatous inflammation. Renal involvement is only seen in a small number of patients. The incidence of renal sarcoidosis varies widely $(0.7 \%-10 \%)$, in part due to varying definitions used in different studies. $^{3}$

Kidney biopsy is often performed to evaluate patients with sarcoidosis presenting with acute kidney injury (AKI). Besides granulomatous interstitial nephritis (GIN), a variety of different histological lesions associated with glomerular involvement in sarcoidosis have been described, including membranous nephropathy, diffuse proliferative or crescentic glomerulonephritis, and focal segmental glomerulosclerosis. This demonstrates the need for further diagnostic evaluation including a kidney biopsy. However, sampling error is fairly common given the focal nature of renal sarcoidosis. Thus, a nondiagnostic kidney biopsy in these patients represents a major diagnostic and therapeutic dilemma, even in the present era.

This paper reports a patient with a history of pulmonary sarcoidosis who presented with AKI. Kidney functions improved with steroid treatment but rapidly deteriorated after completion of the steroid taper. In spite of a nondiagnostic kidney biopsy, the patient was empirically treated with prednisone for presumed renal sarcoidosis. Kidney functions improved within a month of prednisone, although not back to baseline. He continues to be stable on low-dose prednisone. With this case as a background, it is hypothesized that although clinicians often perform a kidney biopsy in patients with sarcoidosis presenting with AKI, it has limited diagnostic utility. A retrospective chart review of patients with sarcoidosis presenting with AKI who underwent a native kidney biopsy was performed to determine the incidence of nondiagnostic kidney biopsies. Those with confirmed diagnosis of sarcoidosis according to the statement of the American Thoracic Society (ATS) were included. ${ }^{1}$ For the purpose of the study, AKI was defined as an increase in serum creatinine of $\geq 0.3 \mathrm{mg} / \mathrm{dL}$ or an increase to $\geq 150 \%-200 \%$ (1.5- to 2-fold) from baseline (Acute Kidney Injury Network [AKIN] criteria stage I). ${ }^{4}$

\section{Case report}

A 63-year-old Caucasian man was diagnosed with pulmonary sarcoidosis. Three months before presentation, he had developed chest pain, shortness of breath, fatigue, and productive cough for which he received multiple antibiotics without improvement. His medical history was significant for hypertension and was taking amlodipine $5 \mathrm{mg}$ once a day. He denied using other over-the-counter medications including nonsteroidal anti-inflammatory drugs. Computed tomography of the chest showed ground glass opacification with a $1.3 \mathrm{~cm} \times 2.1 \mathrm{~cm}$ left upper lobe calcified nodule. Lung biopsy revealed multiple nonnecrotizing granulomas and aggregates of multinucleated giant cells, consistent with sarcoidosis. Symptomatic improvement was noted after he had taken prednisone $40 \mathrm{mg}$ daily for 2 weeks. This was followed by 6 months of prednisone taper. He had normal kidney function with serum creatinine of $0.9 \mathrm{mg} / \mathrm{dL}$ at the time of initial presentation. By the time he was started on prednisone, the serum creatinine had increased to $2.2 \mathrm{mg}$, which then improved to $1.6 \mathrm{mg} / \mathrm{dL}$. Two months after finishing the course of prednisone, his creatinine was found to be $4 \mathrm{mg} / \mathrm{dL}$. He was referred to the nephrology clinic. His serum calcium was $9.3 \mathrm{mg} / \mathrm{dL}$ (normal range $8.6-10.0 \mathrm{mg} / \mathrm{dL}$ ) with an albumin of $4.1 \mathrm{~g} / \mathrm{dL}$ (normal range $3.4-4.8 \mathrm{~g} / \mathrm{dL}$ ). Urine analysis was unremarkable with a protein:creatinine ratio of $30 \mathrm{mg} / \mathrm{g}$ of creatinine (normoalbuminuria $<30 \mathrm{mg} / \mathrm{g}$ ). Renal ultrasound showed normal-sized kidneys with no hydronephrosis, calculi, or calcification. The autoimmune workup, including antinuclear antibodies and antineutrophil cytoplasmic antibodies, was negative. Complement levels were normal and there was a negative viral hepatitis panel. A kidney biopsy was performed to aid the diagnosis.

The biopsy revealed nonspecific findings including mild to moderate focal interstitial fibrosis, but there was no evidence of granulomatous inflammation, calcium-phosphate deposition or immune complex deposition (Figure 1). On electron microscopy, the glomerular basement membrane was of normal thickness and texture without podocyte foot process effacement.

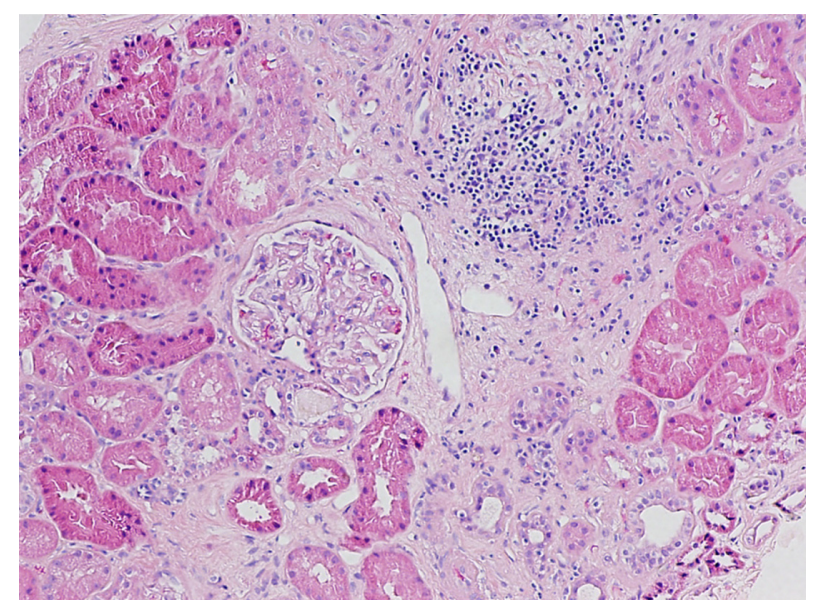

Figure I An area of interstitial fibrosis with mild mononuclear cell inflammation. Note the normal glomerulus.

Notes: Stained with hematoxylin-eosin; magnification $\times 100$ 
Despite the nonspecific kidney biopsy findings, the patient was restarted on prednisone, based on the strong clinical suspicion of renal sarcoidosis. His kidney function improved after restarting the prednisone therapy. Figure 2 shows the progression of serum creatinine before, during, and after prednisone therapy. Serum creatinine improved to $2 \mathrm{mg} / \mathrm{dL}$ within 1 month of treatment, though not to baseline. The patient continued to be stable on low-dose prednisone. Considering the incongruence between the pathological findings and clinical course, and remarkable improvement after treatment with steroids, we conducted a systematic retrospective review of all kidney biopsies done in patients with sarcoidosis and AKI at our institution.

\section{Methods Objectives}

The primary objective was to determine the incidence of nondiagnostic kidney biopsies in patients with biopsy-proven sarcoidosis and AKI. It was also planned to identify the various histological findings seen in this group of patients.

\section{Patient selection}

In this retrospective study, all patients who had native kidney biopsies read at The Ohio State University from January 1, 2003 to December 31, 2009 were identified. Those patients with a diagnosis of sarcoidosis presenting with AKI were included for further review.

\section{Kidney biopsy workup}

Tissue for light microscopy was fixed in $10 \%$ formalin, embedded in paraffin, sectioned $(2-3 \mu \mathrm{m})$, and stained with hematoxylin-eosin, periodic acid-Schiff, Masson's trichrome, and methenamine silver. For direct immunofluorescence, the

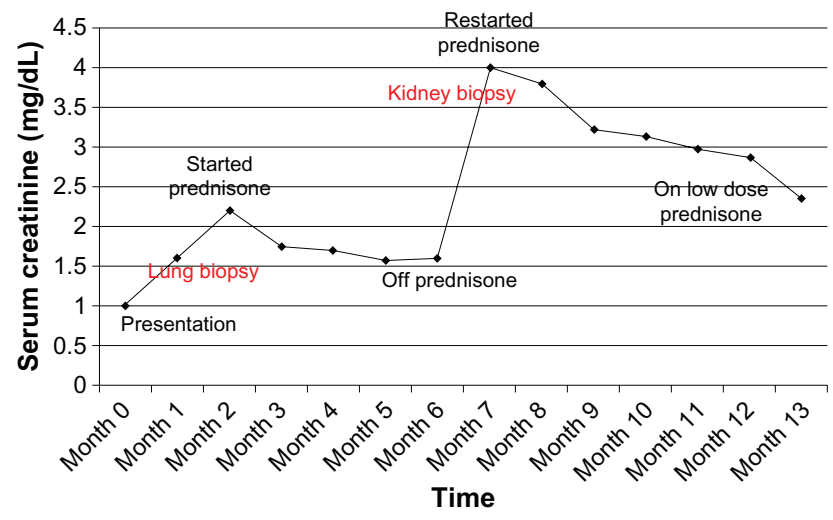

Figure 2 Time course of serum creatinine and prednisone therapy. tissue was placed in optimal cutting temperature compound and snap frozen in liquid nitrogen. Fluorescein isothiocyanate-conjugated primary polyclonal rabbit antihuman antibodies to immunoglobulin (Ig) G, IgA, IgM, C1q, C3, fibrinogen, and $\kappa$ and $\lambda$ light chains (all from Dako Cytomation, Carpinteria, CA) were used on frozen sections. The intensity of staining was graded on a scale from 0 to $3+$ (1+, mild staining; $2+$, moderate staining; $3+$, strong staining; \pm , trace staining). For electron microscopy, tissue was fixed in 3\% buffered glutaraldehyde (pH 7.4) and postfixed in osmium tetroxide. Following routine processing and embedding into Spurr resin, thin sections were cut and contrasted with uranyl acetate and lead citrate. Ultrastructural examination was performed using a Carl Zeiss EM 900 (Carl Zeiss AG, Oberkochen, Germany) transmission electron microscope.

\section{Results}

A total of 2780 native kidney biopsy specimens were read during the study period. Out of these, 21 patients with a history of biopsy-proven sarcoidosis presented with AKI and underwent a native kidney biopsy. Of those, 15 patients $(71 \%)$ were male and six patients $(29 \%)$ were female with a mean age of $55 \pm 14.37$ years. Fifteen patients were Caucasian (71\%) and six (29\%) were African American. Demographics; comorbid conditions; laboratory data, including baseline creatinine and peak serum creatinine; and a 24-hour urine protein excretion are summarized in Table 1.

The histopathological findings of these patients were divided into four groups (Table 2). Only four patients (19\%) showed findings of granulomatous interstitial nephritis (GIN), a histopathological hallmark of renal sarcoidosis. An equal number of patients (19\%) had biopsy findings suggestive of

Table I Patient characteristics

\begin{tabular}{ll}
\hline Age (years) (mean \pm SD) & $\mathbf{5 5} \pm \mathbf{1 4 . 3 7}$ \\
\hline Gender & 15 (7I\%) \\
Male & 6 \\
Female & \\
Race & $15(71 \%)$ \\
$\quad$ Caucasian & 6 \\
African American & \\
Comorbid conditions & $12(57 \%)$ \\
$\quad$ Hypertension & $10(48 \%)$ \\
DM & \\
Laboratory data & $1.05 \pm 0.16$ \\
Baseline Cr (mg/dL) (mean \pm SD) & $2.88 \pm 1.85$ \\
Peak Cr (mg/dL) (Mean \pm SD) & $4.3 \pm 2.33$ \\
Proteinuria (gm/24 hours) (mean \pm SD)
\end{tabular}

Abbreviations: $\mathrm{Cr}$, creatinine; $\mathrm{DM}$, diabetes mellitus; $\mathrm{SD}$, standard deviation. 
Table 2 Histopathological kidney biopsy findings

\begin{tabular}{ll}
\hline Findings & Number of patients $(\mathbf{n}=\mathbf{2 1})$ \\
\hline $\begin{array}{l}\text { Granulomatous interstitial nephritis } \\
\text { (renal sarcoidosis) }\end{array}$ & $4 / 21(19 \%)$ \\
Membranous nephropathy & $4 / 21(19 \%)$ \\
Diabetic nephropathy & $4 / 21(19 \%)$ \\
Nephrocalcinosis & $3 / 21(14 \%)$ \\
Nonspecific changes of chronic & $6 / 21(29 \%)$ \\
kidney injury & \\
\hline
\end{tabular}

Abbreviation: $\mathrm{n}$, number.

either membranous mephropathy or diabetic nephropathy. A third of the patients had nonspecific changes characteristic of chronic kidney injury. Presence of nephrocalcinosis was noted in three patients (14\%).

\section{Discussion}

Sarcoidosis is an idiopathic multisystem illness characterized by granulomatous inflammation, postulated to be an autoimmune response to either an infection or an unknown environmental agent. ${ }^{1-3}$ Diagnosis rests on the demonstration of characteristic noncaseating granulomas in a biopsy specimen of one or more involved organs and clinical exclusion of other important causes of granulomatous inflammation. ${ }^{3,4}$ Worldwide, sarcoidosis is diagnosed most often at about 40 years of age and across all ethnicities, although, in the USA, African Americans have a tenfold greater incidence than Caucasians. ${ }^{5,6}$

Although it can affect any organ system, according to a retrospective registry, involvement of pulmonary, skin, lymphatic, and ocular systems was respectively seen in $95 \%, 15.9 \%, 15.2 \%$, and $11.8 \%{ }^{4,7}$ The true incidence of renal sarcoidosis is hard to determine, in part because of varying definitions. In the aforementioned series of 736 patients, the incidence of renal sarcoidosis was $0.7 \% .{ }^{4,7}$ Although sarcoidosis is primarily a granulomatous inflammation, GIN accounts for only a small proportion of clinically significant kidney failure in patients with sarcoidosis. Instead, deranged vitamin D and calcium metabolism leading to hypercalcemia, hypercalciuria, nephrolithiasis, and nephrocalcinosis are more common problems. ${ }^{8,9}$ In normal subjects, the conversion of 25-hydroxyvitamin D to calcitriol occurs via 1-hydroxylase in the kidney. This is under the physiologic control of parathyroid hormone (PTH), which is suppressed by hypercalcemia. The lack of suppression of calcitriol production in sarcoidosis and other granulomatous diseases is due to PTH-independent extrarenal production of calcitriol from calcidiol by activated mononuclear cells (particularly macrophages) in the lung and lymph nodes. ${ }^{10,11}$ Furthermore, polyuria is often associated with hypercalcemia and hypercalciuria due to antidiuretic hormone dysregulation and can cause hemodynamically mediated AKI. ${ }^{12-14}$ Renal tubular defects, glomerular diseases, mechanical urinary tract disease, and renovascular diseases complete the spectrum of sarcoidosisrelated kidney diseases. ${ }^{3}$

GIN is a rare histologic diagnosis that is present in $0.5 \%-0.9 \%$ of native kidney biopsies and $0.6 \%$ of kidney transplant biopsies. ${ }^{15-17}$ GIN has been associated with medications, infections, sarcoidosis, crystal deposits, paraproteinemia, and Wegener's granulomatosis and can also be idiopathic. Only small series and anecdotal reports of GIN in sarcoidosis are available in the literature..$^{18,19}$

Table 3 Studies evaluating histological findings in patients with renal sarcoidosis

\begin{tabular}{|c|c|c|c|c|c|c|c|}
\hline Published & Study type & $\mathbf{N}$ & $\begin{array}{l}\text { eGFR on presentation } \\
(\mathrm{mL} / \mathrm{min})\end{array}$ & $\begin{array}{l}\text { eGFR at I year } \\
(\mathrm{mL} / \mathrm{min})\end{array}$ & $\begin{array}{l}\text { Kidney } \\
\text { biopsy }\end{array}$ & Histopathological findings & $\begin{array}{l}\text { Median follow-up } \\
\text { (months) }\end{array}$ \\
\hline $\begin{array}{l}\text { Rajakariar } \\
\text { et } \mathrm{al}^{22}\end{array}$ & $\begin{array}{l}\text { Single center } \\
\text { retrospective } \\
\text { case series }\end{array}$ & 39 & $26.8 \pm 14$ & $49.6 \pm 5.2$ & $20 *(51 \%)$ & $\begin{array}{l}\text { TIN: I7/20(86\%) } \\
\text { Granuloma: I6/20 (80\%) } \\
\text { Glomerular diseases with } \\
\text { no TIN: } \\
\text { - Membranous GN: I } \\
\text { - FSGS: I } \\
\text { - Minimal change: I } \\
\text { Intracellular calcifications: } 5\end{array}$ & 84 \\
\hline $\begin{array}{l}\text { Mahevas } \\
\text { et } \mathrm{al}^{23}\end{array}$ & $\begin{array}{l}\text { Multicenter } \\
\text { retrospective } \\
\text { study }\end{array}$ & 47 & $20 \pm 19$ & $47 \pm 19.9$ & $47 *(100 \%)$ & $\begin{array}{l}\text { GIN: } 37 \\
\text { Interstitial nephritis with no } \\
\text { GIN: } 10 \\
\text { Interstitial calcifications: } 8\end{array}$ & 24 \\
\hline
\end{tabular}

Note: *Tubular atrophy and interstitial fibrosis were seen in all patients.

Abbreviations: N, number of patients; eGFR, estimated glomerular filtration rate; MDRD, modification of diet in kidney disease formula; GIN, granulomatous interstitial nephritis; TIN, tubulointerstitial nephritis; FSGS, focal segmental glomerulosclerosis. 
A variety of different histological lesions associated with glomerular involvement in sarcoidosis have been described, including membranous nephropathy, diffuse proliferative or crescentic glomerulonephritis, and focal segmental glomerulosclerosis. The variation demonstrates the importance of kidney biopsy. ${ }^{20,21}$

Few studies have evaluated histological findings in patients with renal sarcoidosis (Table 3). However, patients in these studies already had markedly impaired kidney function (chronic kidney disease stage III) on presentation, presumably due to renal sarcoidosis. There was significant improvement noted in kidney function after prednisone treatment. This might explain why the majority of the study population showed the presence of tubulointerstitial nephritis or $\operatorname{GIN}(80 \%){ }^{22,23}$

Patients with sarcoidosis often present with acute kidney injury. A kidney biopsy is often performed to aid the diagnosis of renal sarcoidosis. As seen in this study, almost half of the patients had nonspecific findings, including diabetic nephropathy, interstitial fibrosis, and tubular atrophy. Thus, the absence of characteristic kidney biopsy findings does not exclude the diagnosis of renal sarcoidosis. This was a retrospective study done at a single institution, which might limit the generalization of our findings. As many of the kidney biopsy specimens were sent from various outside hospitals, the information provided from the referring physicians was relied upon and complete information about the treatment and long term follow-up on these patients was not available.

Corticosteroids are the cornerstone for sarcoidosis treatment, usually started at the equivalent of prednisone $1 \mathrm{mg}$ / $\mathrm{kg}$ /day. ${ }^{21-24}$ There is usually an impressive response but relapses are frequent, particularly when steroids are tapered. These relapses usually respond to reinitiation of steroids, but most patients are left with chronic kidney dysfunction from interstitial fibrosis. ${ }^{21-24}$ Patients who are steroid intolerant or steroid resistant have been treated with cyclophosphamide, infliximab, azathioprine, and mycophenolate mofetil with varying degrees of success. ${ }^{25}$

\section{Conclusion}

In patients with sarcoidosis presenting with AKI, clinicians often rely on kidney biopsy as a diagnostic tool. However, kidney biopsy findings are often nondiagnostic, perhaps because of sampling issues. The absence of kidney biopsy findings characteristic of sarcoidosis does not exclude the possibility of kidney involvement in the disease. The presence of GIN on kidney biopsy, although classic, is uncommon, especially during the early stages. This study underscores the limited diagnostic utility of kidney biopsy in patients with sarcoidosis presenting with AKI. A trial of steroid therapy is warranted in patients with strong clinical suspicion of renal sarcoidosis, even with a nondiagnostic kidney biopsy.

\section{Disclosure}

The authors report no conflicts of interest in this work.

\section{References}

1. Statement on sarcoidosis. Joint statement of the American Thoracic Society, the European Respiratory Society and the World Association of Sarcoidosis and Other Granulomatous Disorders. Am J Respir Crit Care Med. 1999;160(2):736-755.

2. Baughman RP, Lower EE, du Bois RM. Sarcoidosis. Lancet. 2003; 361(9363):1111-1118.

3. Berliner AR, Haas M, Choi MJ. Sarcoidosis: the nephrologist's perspective. Am J Kidney Dis. 2006;48(5):856-870.

4. Mehta RL, Kellum JA, Sha SV, et al. Acute Kidney Injury Network: report of an initiative to improve outcomes in acute kidney injury. Crit Care. 2007;11(2):R31.

5. Walker J, Baran D, Yakub N, Freeman RB. Histoplasmosis with hypercalcemia, kidney failure, and papillary necrosis. Confusion with sarcoidosis. JAMA. 1977;237(13):1350-1352.

6. Thomas K, Hunninghake G. Sarcoidosis. JAMA. 2003;289(24): 3300-3303.

7. James DG. Sarcoidosis. Postgrad Med J. 1984;60(701):234-241.

8. Baughman RP, Teirstein AS, Judson MA, et al. Clinical characteristics of patients in a case control study of sarcoidosis. Am J Respir Crit Care Med. 2001;164(10 Pt 1):1885-1889.

9. Muther R, McCaron D, et al. Kidney manifestations of sarcoidosis. Arch Int Med. 1981;141(5):643-645.

10. Casella FJ, Allon M. The kidney in sarcoidosis. J Am Soc Nephrol. 1993;3(9):1555-1562.

11. Adams JS, Sharma OP, Gacad MA, Singer FR. Metabolism of 25-hydroxyvitamin D3 by cultured pulmonary alveolar macrophages in sarcoidosis. J Clin Invest. 1983;72(5):1856-1860.

12. Adams JS, et al. Isolation and structural identification of 1,25dihydroxyvitamin D3 produced by cultured alveolar macrophages in sarcoidosis. J Clin Endocrinol Metab. 1985;60(5):960-966.

13. Singer FR, Adams JS. Abnormal calcium homeostasis in sarcoidosis. N Engl J Med. 1986;315(12):755-757.

14. Stuart CA, Neelon FA, Lebovitz HE. Disordered control of thirst in hypothalamic-pituitary sarcoidosis. N Engl J Med. 1980; 303(19):1078-1082

15. O’Riordan E, Willert RP, Reeve R, et al. Isolated sarcoid granulomatous interstitial nephritis: review of five cases at one center. Clinical Nephrology. 2001;55(4):297-302.

16. Mignon F, Mery JP, Mougenot B, et al. Granulomatous interstitial nephritis. Adv Nephrol Necker Hosp. 1984;13:219-245.

17. Meehan SM, Josephson MA, Haas M. Granulomatous tubulointerstitial nephritis in the kidney allograft. Am J Kidney Dis. 2000;36(4):E27.

18. Hannedouche T, Grateau G, Noel LH, et al. Renal granulomatous sarcoidosis: report of six cases. Nephrol Dial Transplant. 1990;5(1):18-24.

19. Robson M, Banerjee D, Hopster D, Cairns HS. Seven cases of granulomatous interstitial nephritis in the absence of extrarenal sarcoid. Nephrol Dial Transplant. 2003;18(2):280-284.

20. Toda T, Kimoto S, Nishio Y, Ehara T, Sasaki S. Sarcoidosis with membranous nephropathy and granulomatous interstitial nephritis. Intern Med. 1999;38(11):882-886.

21. Van Uum SH, Cooreman MP, Assmann KJ, Wetzels JF. A 58-year-old man with sarcoidosis complicated by focal crescentic glomerulonephritis. Nephrol Dial Transplant. 1997;12(12):2703-2707. 
22. Rajakariar R, Sharples EJ, Raftery MJ, Sheaff M, Yaqoob MM. Sarcoid tubulo-interstitial nephritis: long-term outcome and response to corticosteroid therapy. Kidney Int. 2006;70(1):165-169.

23. Mahevas M, Lescure FX, Boffa JJ, et al. Renal sarcoidosis: clinical, laboratory, and histologic presentation and outcome in 47 patients. Medicine. 2009;88(2):98-106.
24. Moudgil A, Przygodzki RM, Kher KK. Successful steroid-sparing treatment of kidney limited sarcoidosis with mycophenolate mofetil. Pediatr Nephrol. 2006;21(2):281-285.

25. Ahmed MM, Mubashir E, Dossabhoy NR. Isolated renal sarcoidosis: a rare presentation of a rare disease treated with infliximab. Clin Rheumatol. 2007;26(8):1346-1349.

\section{Publish your work in this journal}

The International Journal of Nephrology and Renovascular Disease is an international, peer-reviewed open-access journal focusing on the pathophysiology of the kidney and vascular supply. Epidemiology, screening, diagnosis, and treatment interventions are covered as well as basic science, biochemical and immunological studies. The journal welcomes original research, clinical studies, reviews \& evaluations, expert opinion and commentary, case reports and extended reports. The manuscript management system is completely online and includes a very quick and fair peerreview system, which is all easy to use. Visit http://www.dovepress.com/ testimonials.php to read real quotes from published authors.

Submit your manuscript here: http://www.dovepress.com/international-journal-of-nephrology-and-renovascular-disease-journal 